\title{
Right ventricular assist device with an oxygenator using extracorporeal membrane oxygenation as a bridge to lung transplantation in a patient with severe respiratory failure and right heart decompensation
}

\author{
Dong Kyu Oh${ }^{1}$, Tae Sun Shim ${ }^{1}$, Kyung-Wook Jo', Seung-II Park², Dong Kwan Kim², Sehoon Choi², \\ Geun Dong Lee ${ }^{2}$, Sung-Ho Jung ${ }^{2}$, Pil-Je Kang ${ }^{2}$, Sang-Bum Hong ${ }^{1}$ \\ Departments of ${ }^{1}$ Pulmonary and Critical Care Medicine and ${ }^{2}$ Thoracic and Cardiovascular Surgery, Asan Medical Center, University of Ulsan College of \\ Medicine, Seoul, Korea
}

Right heart decompensation is a fatal complication in patients with respiratory failure, particularly in those transitioned to lung transplantation using veno-venous extracorporeal membrane oxygenation (V-V ECMO). In these patients, veno-arterial (V-A ECMO) or veno-arterialvenous extracorporeal membrane oxygenation (V-AV ECMO) is used to support both cardiac and respiratory function. However, these processes may increase the risk of device-related complications such as bleeding, thromboembolism, and limb ischemia. In the present case, a 64-year-old male patient with idiopathic pulmonary fibrosis developed respiratory failure and commenced treatment with V-V ECMO as a bridge to lung transplantation. Unfortunate$l_{y}$, the patient developed right heart decompensation and required both cardiac and respiratory support during treatment with V-V ECMO. Instead of adding arterial cannulation, he was switched to a novel configuration, a right ventricular assist device with an oxygenator (OxyRVAD) using ECMO, with drainage cannulation from the femoral vein and return cannulation to the main pulmonary artery. The patient was successfully bridged to lung transplantation without serious complications after 10 days of Oxy-RVAD support. To the best of our knowledge, this is an extreme rare and challenging case of Oxy-RVAD using ECMO in a patient successfully bridged to lung transplantation.

Key Words: extracorporeal membrane oxygenation; heart-assist devices; heart failure; lung transplantation; respiratory insufficiency; right heart bypass

Lung transplantation has become standard-of-care for patients with nonmalignant end-stage lung disease [1]. However, increasing wait time and significant mortalities while on the wait list continues to be an issue, as the need for donor lungs far exceeds their availability [2]. In such patients, veno-venous extracorporeal membrane oxygenation (V-V ECMO) which supports respiratory function, may be considered as a bridge to lung transplantation [3].

Unfortunately, right heart decompensation is a known complication of V-V ECMO and causes hemodynamic instability [4]. Because this potentially-fatal problem requires both cardiac and respiratory support, immediate interventions are required. These can include the

\section{Case Report}

Received: December 29, 2018

Revised: February 26, 2019

Accepted: February 26, 2019

Corresponding author

Sang-Bum Hong

Department of Pulmonary and

Critical Care Medicine, Asan Medical

Center, University of Ulsan College

of Medicine, 88 Olympic-ro 43-gil,

Songpa-gu, Seoul 05505, Korea

Tel: +82-2-3010-3893

Fax: +82-2-3010-6968

E-mail: hongsangbum@gmail.com

Copyright (c) 2020 The Korean Society of Critical Care Medicine

This is an Open Access article distributed under the terms of Creative Attributions Non-Commercial License (https://

creativecommons.org/li-censes/by-nc/4.0/) which permits unrestricted noncommercial use, distribution, and reproduction in any medium, provided the original work is properly cited. 
addition of arterial cannulation and switching to veno-arterial (V-A ECMO) or veno-arterial-venous ECMO (V-AV ECMO) [4]. However, these configurations may increase the risk of device-related complications such as bleeding, thromboembolism, and limb ischemia and may interfere with early rehabilitation [5].

Herein, we report our extraordinary experience with a novel configuration, a right ventricular assist device with an oxygenator (Oxy-RVAD) using ECMO, in a patient with respiratory failure who developed right heart decompensation during treatment with V-V ECMO. The patient was successfully bridged to lung transplantation, without serious complications, after 10 days of Oxy-RVAD support.

\section{CASE REPORT}

A 64-year-old male (height, $168 \mathrm{~cm}$; weight, $57 \mathrm{~kg}$; body surface area, $1.63 \mathrm{~m}^{2}$ ) was admitted to Asan Medical Center due to acute exacerbation of idiopathic pulmonary fibrosis. $\mathrm{He}$ was treated with broad-spectrum antibiotics and high-dose corticosteroids including pulse therapy with methylprednisolone $500 \mathrm{mg} /$ day for 3 consecutive days. However, the patient's condition rapidly deteriorated and, unfortunately, he was intubated and transferred to the intensive care unit (ICU) 13 days after hospital admission. The bedside transthoracic echocardiography and the blood level of brain natriuretic peptide (BNP) performed at the time of ICU admission revealed no evidence of resting pulmonary hypertension and right heart decompensation (peak tricuspid valve regurgitation velocity, $2.5 \mathrm{~m} / \mathrm{sec}$; tricuspid lateral annulus doppler tissue imaging tricuspid annular peak systolic velocity (S'), $15.9 \mathrm{~cm} / \mathrm{sec}$; and BNP, $55 \mathrm{pg} / \mathrm{ml}$ ) (Table 1).

Because the patient was on a wait list for lung transplantation, we decided to support him with V-V ECMO as a bridge to lung transplantation. Initial V-V ECMO was established the day after ICU admission using a CAPIOX EBS Integrated LX circuit (Terumo Cardiovascular Systems Corp., Tokyo, Japan) which includes an oxygenator and centrifugal pump. After administering 3,000 units of heparin intravenously, cannulation was performed at ICU bedside using the Seldinger technique under ultrasound guidance. A 24-Fr cannula (RMI; Edwards Lifesciences, Irvine, CA, USA) was inserted into the right femoral vein to drain blood from the inferior vena cava. A 17Fr cannula (Bio-Medicus; Medtronic Inc., Minneapolis, MN, USA) was inserted into the right internal jugular vein to return blood to the right atrium. The initial ECMO flow rate was 3.9 $\mathrm{L} / \mathrm{min}$ with a fraction of inspired oxygen $\left(\mathrm{FiO}_{2}\right)$ of $100 \%$ and a gas sweep of $4.0 \mathrm{~L} / \mathrm{min}$. The initial fraction of recirculated blood was 56\%, and was measured using ELSA Monitor (Transonic, Ithaca, NY, USA). Because of the high fraction of recirculated blood, we withdrew the drainage cannula by $5 \mathrm{~cm}$ and increased the distance between drainage and return cannulae from $4 \mathrm{~cm}$ to $9 \mathrm{~cm}$ to reduce recirculation (Figure 1).

A percutaneous dilatational tracheostomy was performed on ECMO day 4, but a bleeding complication occurred at the tracheostomy site. Systemic heparinization was stopped for 24 hours and a topical treatment with 1:1,000 epinephrinesoaked gauze was applied. No systemic hemostatic agents

Table 1. Serial changes in clinical parameters

\begin{tabular}{|c|c|c|c|c|c|}
\hline Variable & V-VECMO & Oxy-RVAD & After Oxy-RVAD & Transplant & After transplant \\
\hline ICU day & 2 & 20 & 24 & 30 & 45 \\
\hline \multicolumn{6}{|l|}{ Echocardiography } \\
\hline Peak TR velocity (m/sec) & 2.5 & 3.7 & 3.3 & Not performed & 2.8 \\
\hline Tricuspid lateral annulus DTI S' (cm/sec) & 15.9 & 6.2 & 7.2 & Not performed & 9.9 \\
\hline \multicolumn{6}{|l|}{ Laboratory finding } \\
\hline BNP (pg/ml) & 55 & 1,131 & 474 & 297 & 228 \\
\hline AST/ALT (IU/L) & $29 / 34$ & $41 / 32$ & $21 / 22$ & $27 / 23$ & $24 / 46$ \\
\hline \multicolumn{6}{|l|}{ Medication } \\
\hline Norepinephrine ( $\mu \mathrm{g} / \mathrm{kg} / \mathrm{min})$ & 0.02 & 0.08 & Not administered & Not administered & Not administered \\
\hline Vasopressin (units/min) & 0.02 & 0.04 & Not administered & Not administered & Not administered \\
\hline Epinephrine ( $\mu \mathrm{g} / \mathrm{kg} / \mathrm{min})$ & Not administered & 0.10 & Not administered & Not administered & Not administered \\
\hline
\end{tabular}

V-V ECMO: veno-venous extracorporeal membrane oxygenation; Oxy-RVAD: right ventricular assist device with an oxygenator; ICU: intensive care unit; TR: tricuspid valve regurgitation; DTI: doppler tissue imaging; $S^{\prime}$ : tricuspid lateral annulus doppler tissue imaging tricuspid annular peak systolic velocity; BNP: brain natriuretic peptide; AST: aspartate transaminase; ALT: alanine transaminase. 

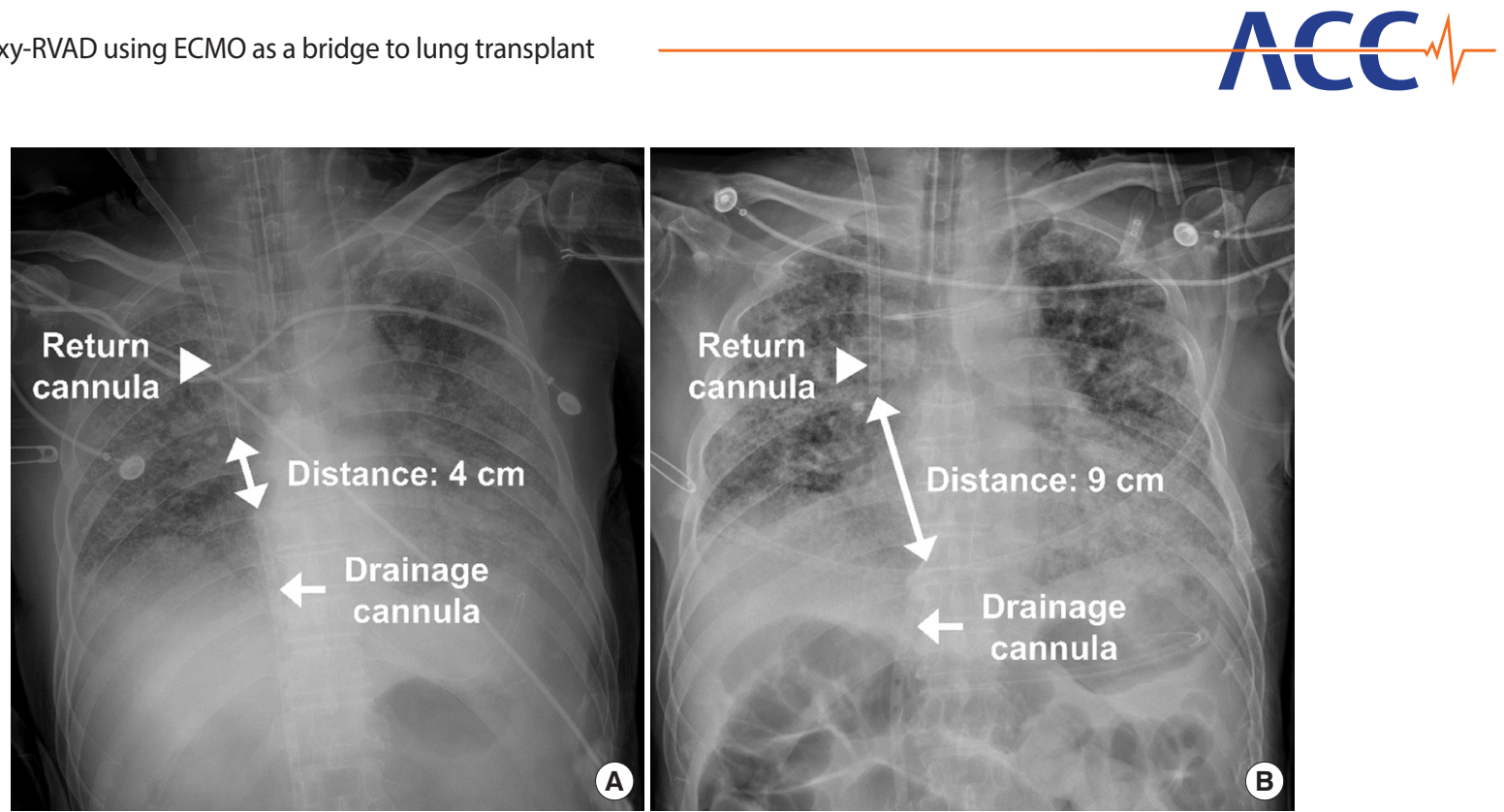

Figure 1. Chest radiographs showing initial position of drainage and return cannulae $(A)$ and those after adjustment due to recirculation (B). Note that the increased distance between drainage and return cannulae after adjustment.

were administered. Unfortunately, blood clots appeared within the oxygenator membrane on ECMO day 5 . We therefore decided to change the ECMO circuit, including the oxygenator membrane and centrifugal pump. A second V-V ECMO was established on ECMO day 5 using a QUADROX PLS System (Maquet Cardiopulmonary AG, Rastatt, Germany). An ECMO flow rate of $4.0 \mathrm{~L} / \mathrm{min}$ was achieved with a $\mathrm{FiO}_{2}$ of $100 \%$ and a gas sweep of $4.0 \mathrm{~L} / \mathrm{min}$. The fraction of recirculated blood was $43 \%$ at the time of EMCO circuit change.

The patient was fully awakened and actively rehabilitated up to standing by participation in our rehabilitation program. However, at ECMO day 17, patient's right heart function began to worsen (Table 1). The echocardiography revealed Dshaped left ventricle with dilated right ventricle and newly developed resting pulmonary hypertension with right ventricular dysfunction (peak tricuspid valve regurgitation velocity, $3.7 \mathrm{~m} / \mathrm{sec}$; tricuspid lateral annulus doppler tissue imaging $\mathrm{S}$, $6.2 \mathrm{~cm} / \mathrm{sec}$ ). The blood level of BNP also increased to 1,131 $\mathrm{pg} / \mathrm{ml}$. Inotropes such as epinephrine were immediately applied, but without improvement in right heart decompensation. As the patient's condition continued to deteriorate despite all medical treatments, we decided to switch his configuration to a Oxy-RVAD using ECMO to enhance gas exchange, improve hemodynamic stability, and facilitate rehabilitation.

At ECMO day 19, the patient was transferred to the operating room and underwent configuration switching to Oxy-RVAD using ECMO, with drainage cannulation from the left femoral vein and return cannulation to the main pulmonary artery (Figure 2). Under general anesthesia, we performed a $5-\mathrm{cm}$

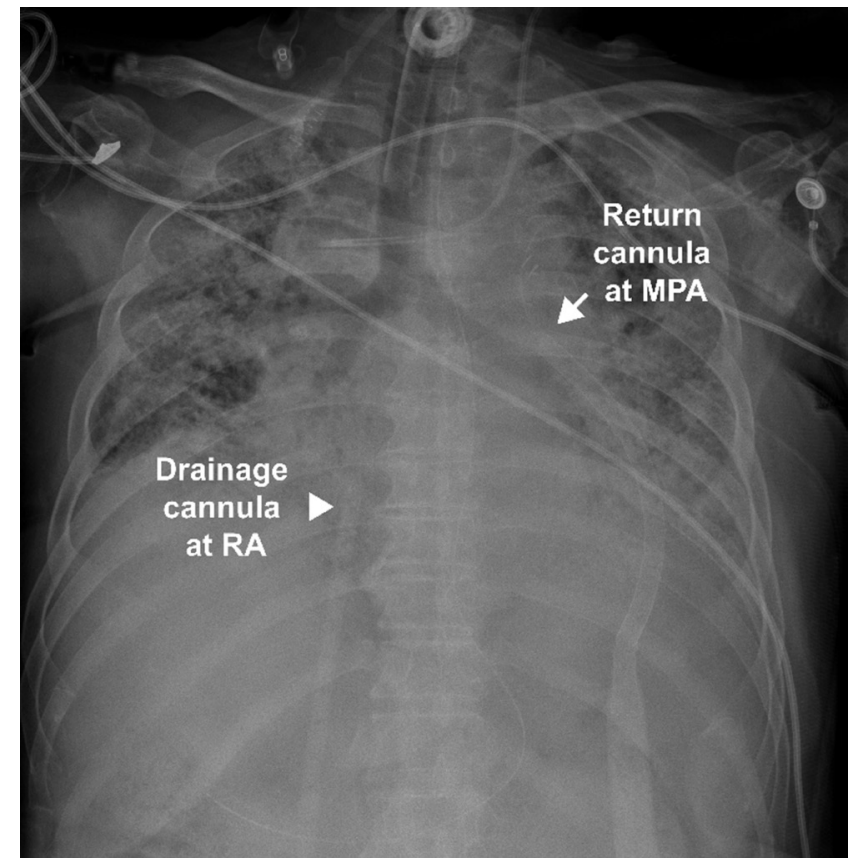

Figure 2. A chest radiograph showing drainage cannula at right atrium (RA) and return cannula at main pulmonary artery (MPA) in right ventricular assist device with an oxygenator support.

sized left anterior thoracotomy at the second intercostal space. A 22-Fr cannula (DLP, Medtronic Inc.) was inserted and endto-side anastomosed to the main pulmonary artery (Figure 3 ). Because the drain cannula at the right femoral vein was already used for 19 days and a catheter-related infection could not be excluded, a new 24-Fr cannula (RMI, Edwards Lifesciences) was inserted into the left femoral vein, and the pre- 


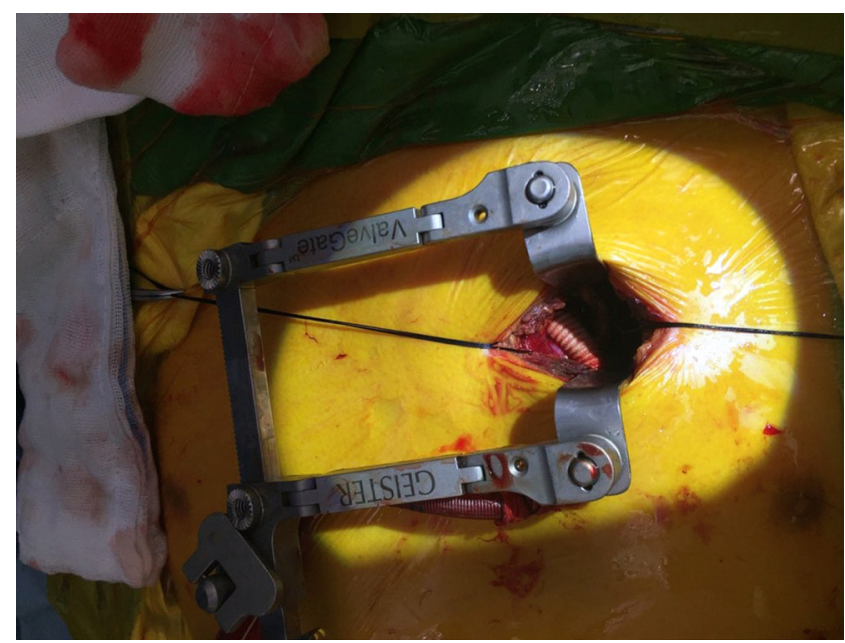

Figure 3. A clinical photograph showing end-to-side anastomosis of return cannula to main pulmonary artery in right ventricular assist device with an oxygenator support.

vious one on the contralateral side was removed. The patient was then transferred to the ICU and monitored closely. At the time of ICU transfer, we achieved an ECMO flow rate of $3.5 \mathrm{~L} /$ $\mathrm{min}$, with a $\mathrm{FiO}_{2}$ of $100 \%$ and a gas sweep of $4.0 \mathrm{~L} / \mathrm{min}$. Immediately after switching the configuration to Oxy-RVAD using ECMO, the partial pressure of oxygen in arterial blood $\left(\mathrm{PaO}_{2}\right)$ increased from $77.9 \mathrm{mmHg}$ to $514.0 \mathrm{mmHg}$ under similar ECMO and ventilator settings, suggesting improved efficacy of ECMO and a decreased fraction of recirculated blood.

After 4 days from Oxy-RVAD support, a follow-up echocardiography was performed (Table 1). Although the resting pulmonary hypertension and global right ventricular hypokinesia were still present, both were improved (peak tricuspid valve regurgitation velocity, $3.3 \mathrm{~m} / \mathrm{sec}$; tricuspid lateral annulus doppler tissue imaging $S^{\prime}: 7.2 \mathrm{~cm} / \mathrm{sec}$ ). The right ventricle size was decreased to $26 \mathrm{~mm}$ and the D-shaped left ventricle was disappeared. The blood level of BNP also decreased to $474 \mathrm{pg} / \mathrm{ml}$. The patient was actively rehabilitated with Oxy-RVAD support and successfully bridged to lung transplantation without serious complication at ECMO day 29, after 10 days from Oxy-RVAD support. The patient was weaned from Oxy-RVAD upon lung transplantation and successfully discharged from the ICU at postoperative day 18 .

\section{DISCUSSION}

V-V ECMO may be considered in patients with severe respiratory failure, particularly those awaiting lung transplantation [3]. However, right heart decompensation often occurs and causes hemodynamic instability which requires both cardiac and respiratory supports during treatment with V-V ECMO [4]. There are several current medical treatments for right heart decompensation including fluid optimization, inotropes, adjustment of ventilator settings, and inhaled nitric oxide [4]. However, these may not be sufficient for patients with hemodynamic instability and, in such patients, mechanical support with V-A or V-AV ECMO may be required $[4,5]$.

Adding arterial cannulation to V-A or V-AV ECMO, which is usually accessed peripherally via the femoral artery at bedside, contributes to hemodynamic stability by providing cardiopulmonary support $[4,5]$. However, like a double-edged sword, it may increase the risk of device-related complications such as bleeding, thromboembolisms, and limb ischemia. Moreover, the peripherally-infused retrograde blood flow into the aorta may also cause catastrophic complications including intracardiac thrombosis and upper body ischemia [5]. In addition, peripherally-inserted arterial cannulation within the inguinal area may interfere with early rehabilitation which is important for patients awaiting lung transplantation, in order to maintain their candidacy [6].

On the other hand, RVAD and Oxy-RVAD seem to provide a more physiological solution for right heart decompensation as these procedures maintain anterograde and normal transpulmonary blood flow $[7,8]$. Furthermore, Noly et al. [8] reported that RVAD is associated with significantly fewer thromboembolic complications than V-A ECMO. In addition, as the ECMO with central or upper body configuration has strength in rehabilitation compared to peripherally inserted V-A ECMO [9], the Oxy-RVAD using ECMO which has return cannula at main pulmonary artery also has the advantage of allowing patients to get out of bed and participate in physical therapy by virtue of the absence of arterial cannula at inguinal area. $\mathrm{Al}$ though there are reports that RVAD may increase pulmonary arterial pressure, potentially causing pulmonary hemorrhage in patients with severe pulmonary hypertension [10], these reports are mostly limited to patients with idiopathic pulmonary arterial hypertension which has a different pathophysiology and clinical manifestation than pulmonary hypertension in patients with chronic lung disease. Few patients with chronic lung disease are included among those with severe pulmonary hypertension [11]; therefore, we assume that pulmonary hemorrhages related to RVAD would be identified lessfrequently in these patients.

In summary, we report on our extraordinary experience with Oxy-RVAD using ECMO in a patient with both severe respiratory failure and right heart decompensation. The patient was actively rehabilitated and successfully bridged to lung trans- 
plantation without serious complications after 10 days of OxyRVAD support. To the best of our knowledge, this is an extreme rare and challenging case of Oxy-RVAD using ECMO which was successfully bridged to lung transplantation. This technique may therefore be beneficial to other patients with similar conditions. In addition, the issues related to possible complications of Oxy-RVAD, such as increased pulmonary arterial pressure and pulmonary hemorrhage, also need to be addressed in future studies. However, Oxy-RVAD with ECMO support may provide a successful bridge to lung transplantation may be achieved in a patient with severe respiratory failure and right heart decompensation.

\section{CONFLICT OF INTEREST}

No potential conflict of interest relevant to this article was reported.

\section{ORCID}

$\begin{array}{ll}\text { Dong Kyu Oh } & \text { https://orcid.org/0000-0002-7511-9634 } \\ \text { Tae Sun Shim } & \text { https://orcid.org/0000-0001-6653-816X } \\ \text { Kyung-Wook Jo } & \text { https://orcid.org/0000-0002-5949-248X } \\ \text { Seung-Il Park } & \text { https://orcid.org/0000-0002-8729-0498 } \\ \text { Dong Kwan Kim } & \text { https://orcid.org/0000-0003-1984-0352 } \\ \text { Sehoon Choi } & \text { https://orcid.org/0000-0002-9961-9289 } \\ \text { Geun Dong Lee } & \text { https://orcid.org/0000-0003-1890-7455 } \\ \text { Sung-Ho Jung } & \text { https://orcid.org/0000-0002-3699-0312 } \\ \text { Pil-Je Kang } & \text { https://orcid.org/0000-0002-7587-0911 } \\ \text { Sang-Bum Hong } & \text { https://orcid.org/0000-0003-2737-7695 }\end{array}$

\section{AUTHOR CONTRIBUTIONS}

Conceptualization \& Data curation: all authors. Formal analysis, Methodology, Project administration, \&Visualization: DKO, SBH. Writing-original draft: DKO, SBH. Writing-review \& editing: all authors.

\section{REFERENCES}

1. Kotloff RM, Thabut G. Lung transplantation. Am J Respir Crit Care Med 2011;184:159-71.

2. Valapour M, Skeans MA, Heubner BM, Smith JM, Schnitzler MA, Hertz MI, et al. OPTN/SRTR 2012 annual data report: lung. Am J Transplant 2014;14 Suppl 1:139-65.

3. Chiumello D, Coppola S, Froio S, Colombo A, Del Sorbo L. Extracorporeal life support as bridge to lung transplantation: a systematic review. Crit Care 2015;19:19.

4. Bunge JJ, Caliskan K, Gommers D, Reis Miranda D. Right ventricular dysfunction during acute respiratory distress syndrome and veno-venous extracorporeal membrane oxygenation. J Thorac Dis 2018;10(Suppl 5):S674-S82.

5. Makdisi G, Wang IW. Extra corporeal membrane oxygenation (ECMO) review of a lifesaving technology. J Thorac Dis 2015, 7:E166-76

6. Hoffman M, Chaves G, Ribeiro-Samora GA, Britto RR, Parreira VF. Effects of pulmonary rehabilitation in lung transplant candidates: a systematic review. BMJ Open 2017;7(2):e013445.

7. Mohite PN, Sabashnikov A, De Robertis F, Popov AF, Simon AR. Oxy-RVAD: rescue in pulmonary complications after LVAD implantation. Perfusion 2015;30:596-9.

8. Noly PE, Kirsch M, Quessard A, Leger P, Pavie A, Amour J, et al. Temporary right ventricular support following left ventricle assist device implantation: a comparison of two techniques. Interact Cardiovasc Thorac Surg 2014;19:49-55.

9. Biscotti M, Bacchetta M. The "sport model": extracorporeal membrane oxygenation using the subclavian artery. Ann Thorac Surg 2014;98:1487-9.

10. Gregoric ID, Chandra D, Myers TJ, Scheinin SA, Loyalka P, Kar B. Extracorporeal membrane oxygenation as a bridge to emergency heart-lung transplantation in a patient with idiopathic pulmonary arterial hypertension. J Heart Lung Transplant 2008;27:466-8.

11. Seeger W, Adir Y, Barberà JA, Champion H, Coghlan JG, Cottin $\mathrm{V}$, et al. Pulmonary hypertension in chronic lung diseases. J Am Coll Cardiol 2013;62(25 Suppl):D109-16. 